\title{
The Optimal Antenna Layout for Maximum Ergodic Capacity of MISO Beamforming System
}

\author{
Qianya Wang and Hongwen Yang \\ School of Information and Communication Engineering, Beijing University of Posts and Telecommunications (BUPT), \\ Beijing 100876, China
}

Correspondence should be addressed to Qianya Wang; wangqianya.bupt@gmail.com

Received 21 June 2013; Revised 14 September 2013; Accepted 12 October 2013

Academic Editor: Demosthenes Vouyioukas

Copyright (C) 2013 Q. Wang and H. Yang. This is an open access article distributed under the Creative Commons Attribution License, which permits unrestricted use, distribution, and reproduction in any medium, provided the original work is properly cited.

\begin{abstract}
The performance of the multiantenna systems can be deeply affected by the antenna layout, that is, the placement of the antenna elements in a given area. This paper focuses on the optimal antenna layout which can maximize the ergodic capacity of a multiinput single-output (MISO) beamforming system. Since the optimization problem cannot be solved analytically, we use simulated annealing (SA) algorithm to search for the optimal antenna layout. The results show that the optimal antenna layout depends highly on the power azimuth spectrum (PAS) distribution and power elevation spectrum (PES) distribution. In the three-dimensional (3D) space uniformly scattering environment, the optimal antenna layout will locate the antenna elements on the surface of a sphere. When the scattering is concentrated in a two-dimensional (2D) plane, the circular layout is the optimal and finally, when there is no scattering (single plane wave), the optimal layout is the nonuniform linear array.
\end{abstract}

\section{Introduction}

multiple-input multiple-output (MIMO) systems have received significant attention for their capability of increasing channel capacity and improving the transmission reliability $[1,2]$. Beamforming $(\mathrm{BF})$ is one particular way of utilizing multiple antenna elements $[3,4]$. In a beamforming antenna array, the radiated electromagnetic field can be patterned to have high antenna gain in desired direction by controlling the phase and amplitude of the signal field at each antenna element. Antenna beamforming has found a diverse range of applications due to its simplicity and effectiveness [5-8].

For a practical system, it is typical that multiple antenna elements have to be installed in a limited space area. The constrained space area will inevitably introduce the correlation between antenna elements and such correlation will affect the performance of multiantenna system. There are already many published works discussing the relation between the antenna correlation and the performance of multiple antenna systems in terms of ergodic capacity, outage capacity, bit error rate, and so forth [9-14]. However, to our knowledge, there are relatively few reports [15-22] on the relation between the multiple antenna system performance and the antenna layout, that is, the placement of the antenna elements. It is well known that, given the power azimuth spectrum (PAS) distribution and power elevation spectrum (PES) distribution [23], the performance of the multiantenna system is completely determined by the antenna spatial correlation matrix with its entries determined by the relative distance of the antenna elements. Hence, it is clear that the performance of the multiple antenna system is a function of antenna layout. In case of 2-dimensional (2D) deployment of multiple antenna elements and uniform PAS, it is reported $[19,20]$ that the optimal antenna layout which can maximize MIMO capacity is the near circular array, but both of them considered a propagation model including PAS only. For short-range communications, [21] has investigated the optimal antenna placement of $2 \times 2$ MIMO taken into consideration the joint effects of path loss and phase difference. In [22], empirical results for the effects of antenna placement on vehicle-tovehicle link performance in vehicular ad hoc networks have been reported.

This paper focuses on the 3-dimensional (3D) deployments of multiple transmit antenna elements. In particular, 
we are interested in the optimal antenna elements placement which can maximize the ergodic capacity of a multipleinput single-output (MISO) BF system. Since it is difficult (if not impossible) to find the optimal solution analytically, simulated annealing (SA) algorithm [24-26] is used to search for the optimal antenna layouts for different PAS and PES. The results show that the optimal antenna layout is highly dependent on the PAS and PES distribution. In the $3 \mathrm{D}$ space uniformly scattering environment, the layout with antenna elements located on a surface of sphere will obtain the best ergodic capacity. When the spherical power spectrum (SPS) [23] degrades to 2D distribution (with only PAS or PES), the circular layout is the optimal. While there is no angle spreading, the nonuniform linear array is the best.

The rest of the paper is organized as follows. The next section introduces the system model including the 3D spatial correlation model and the ergodic capacity expressed as the function of eigenvalues of antenna correlation matrix. In Section 3, we use the simulated annealing to search for the optimal antenna layouts for some representative PAS and PES. Section 4 concludes the paper.

Throughout the paper, the following notations are adopted. All the matrices and the vectors are denoted by boldfaced capital letters and boldfaced small letters, respectively. $(\cdot)^{H}$ and $(\cdot)^{T}$ denote the complex conjugate transpose and transpose operator, respectively. $|\cdot|$ is the absolute value and $\|\cdot\|$ is the two-norm operator. $E\{\cdot\}$ represents the expectation. $\mathrm{CN}(0, \mathbf{A})$ denotes a complex circular-symmetric Gaussian random vector with zero mean and variance matrix $\mathbf{A}$.

\section{System Model}

2.1. MISO Beamforming System. Consider an MISO system shown in Figure 1(a) with $L$ transmit antennas and a single receive antenna, where $d_{\max }$ is the maximum allowable separation distance between any two antenna elements and $d$ is the distance between the transmitter and receiver. For most applications, $d$ is much larger than $d_{\max }$. Suppose that all the antennas are mounted with the same orientation in 3D space, as shown in Figure 1(a); we can reasonably assume that the path loss, shadowing, and the antenna gain (due to the antenna pattern) are the same for all antenna elements.

Based on the principle of reciprocity, the characteristics of an antenna array will keep the same whatever it is used for transmitting or receiving. Consider the transmit antenna array shown in Figure 1(b); the electromagnetic waves (rays) will depart from the array in different directions, due to the scattering in the environment. For each ray, we use $\alpha$ to denote the elevation and $\beta$ the azimuth. The total energy is distributed along these directions and the distribution is denoted, respectively, by $P_{e}(\alpha)$ and $P_{a}(\beta)$, namely, the power azimuth spectrum (PAS) and the power elevation spectrum (PES) $[14,27]$.

The MISO channel is modeled as a $L \times 1$ vector $\mathbf{h}=$ $\left(h_{1}, h_{2}, \ldots, h_{L}\right)^{T}$, where $h_{l}(l=1,2, \ldots, L)$ is the channel coefficient from the $l$ th transmit antenna to the receive antenna. Based on this assumption, we can model $\mathbf{h}$ as a zero

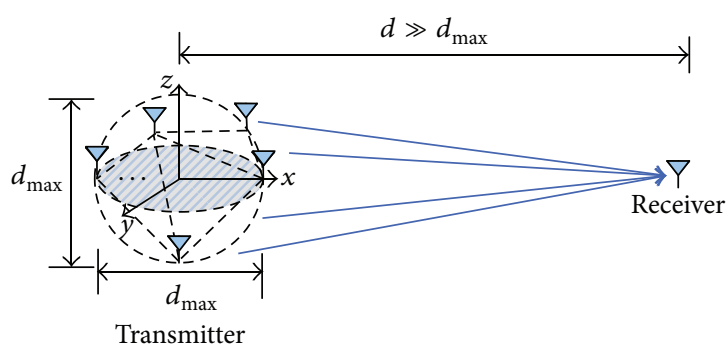

(a) MISO system

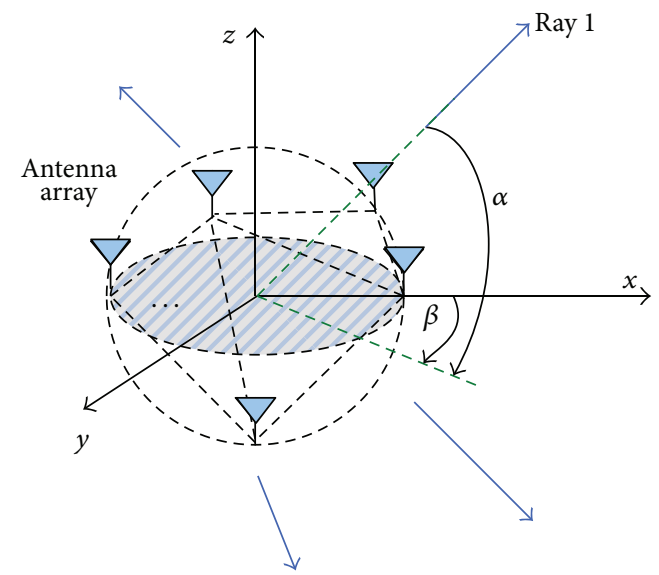

(b) Electromagnetic waves (rays) departing from different directions. For each ray, $\alpha$ denotes the elevation and $\beta$ denotes the azimuth

FIGURE 1: System model. mean complex circular-symmetric Gaussian vector $\mathrm{CN}(0, \mathbf{R})$, where $\mathbf{R}$ is the transmit antenna correlation matrix defined as

$$
\mathbf{R}=E\left[\mathbf{h h}^{H}\right] .
$$

The received signal at the receiver is

$$
r=\mathbf{h}^{T} \mathbf{w} s+n,
$$

where $n$ is the zero mean complex additive Gaussian white noise (AWGN) with variance $N_{0}$ and $s$ is the scalar data symbol with $E\left[|s|^{2}\right]=E_{s}$. Before transmission on antenna $l$, the symbol is weighted by a complex number $w_{l}$. The weights for all transmit antennas can be collected into a $L \times 1 \mathrm{BF}$ vector $\mathbf{w}=\left(w_{1}, w_{2}, \ldots, w_{L}\right)^{T}$.

Assuming perfect channel state information (CSI) at the transmitter, then the optimal BF weighting vector is given by

$$
\mathbf{w}=\frac{\mathbf{h}^{H}}{\|\mathbf{h}\|},
$$

and the instantaneous signal to noise ratio (SNR) at the output of receiver is given by

$$
\gamma=\frac{E_{s}}{N_{0}}\|\mathbf{h}\|^{2}=\gamma_{0}\|\mathbf{h}\|^{2},
$$

where $\gamma_{0}=E_{s} / N_{0}$. 
2.2. Correlation between Antenna Elements. The elements of $\mathbf{R}$ defined in (1) are the correlation coefficient of a pair of transmit antenna elements. With the classical multipath model [28-31], for any two transmit antennas located at $\mathbf{p}=$ $(x, y, z)$ and $\mathbf{p}^{\prime}=\left(x^{\prime}, y^{\prime}, z^{\prime}\right)$, the $3 \mathrm{D}$ spatial correlation is given by

$$
\begin{gathered}
R\left(\mathbf{p}, \mathbf{p}^{\prime}\right) \\
=\int_{-\pi / 2}^{\pi / 2} \int_{-\pi}^{\pi} \exp \left\{j \frac { 2 \pi } { \lambda } \left[\left(x-x^{\prime}\right) \cos \alpha \cos \beta\right.\right. \\
+\left(y-y^{\prime}\right) \cos \alpha \sin \beta \\
\left.\left.+\left(z-z^{\prime}\right) \sin \alpha\right]\right\} \\
\times P_{e}(\alpha) P_{a}(\beta) \mathrm{d} \alpha \mathrm{d} \beta,
\end{gathered}
$$

where $\lambda$ is the wavelength and $P_{e}(\alpha)$ and $P_{a}(\beta)$ are the PAS and PES with $\alpha$ and $\beta$ defined in Figure 1(b).

An antenna layout is a set of $L$ antenna positions: $\mathbf{P}=$ $\left\{\mathbf{p}_{1}, \mathbf{p}_{2}, \ldots, \mathbf{p}_{L}\right\}$ where $\mathbf{p}_{l}=\left(x_{l}, y_{l}, z_{l}\right)$ is the $3 \mathrm{D}$ Cartesian coordinates of the $l$ th antenna element. Given the PAS and PES, the antenna correlation matrix $\mathbf{R}$ can be completely determined by $\mathbf{P}$ through (5).

2.3. Ergodic Capacity. The instantaneous capacity, in nats per symbol, for MISO BF system is given by

$$
C=\ln (1+\gamma)
$$

where $\gamma$ is defined in (4). The ergodic capacity [31] is defined as the expectation of (6), which is

$$
\bar{C}=E[\ln (1+\gamma)]=\int_{0}^{\infty}[\ln (1+x)] f_{\gamma}(x) \mathrm{d} x,
$$

where $f_{\gamma}(x)$ is the probability density function (p.d.f.) of $\gamma$.

If the correlation matrix $\mathbf{R}$ is known, then $f_{\gamma}(x)$ is given by $[32,33]$ as

$$
f_{\gamma}(x)=\sum_{l=1}^{L} \frac{\sigma_{l}^{L-2}}{\prod_{k \neq l}\left(\sigma_{k}-\sigma_{l}\right)} \exp \left(-\frac{x}{\gamma_{0} \sigma_{l}}\right),
$$

where $0<\sigma_{1}<\cdots<\sigma_{l}<\cdots<\sigma_{L}$ are the distinct eigenvalues of R. Substituting (8) into (7), the ergodic capacity is given as

$$
\begin{aligned}
\bar{C} & =\sum_{l=1}^{L} \frac{\sigma_{l}^{L-2}}{\prod_{k \neq l} \sigma_{k}-\sigma_{l}} \int_{0}^{\infty} \ln (1+x) \exp \left(-\frac{x}{\gamma_{0} \sigma_{l}}\right) \mathrm{d} x \\
& =\sum_{l=1}^{L} \frac{\sigma_{l}^{L}}{\prod_{k \neq l}\left(\sigma_{k}-\sigma_{l}\right)} \exp \left(\frac{1}{\gamma_{0} \sigma_{l}}\right) E_{1}\left(\frac{1}{\gamma_{0} \sigma_{l}}\right),
\end{aligned}
$$

where $E_{1}(x)$ is the exponential integral function, defined as

$$
E_{1}(x)=\int_{x}^{\infty} \frac{e^{-t}}{t} \mathrm{~d} t
$$

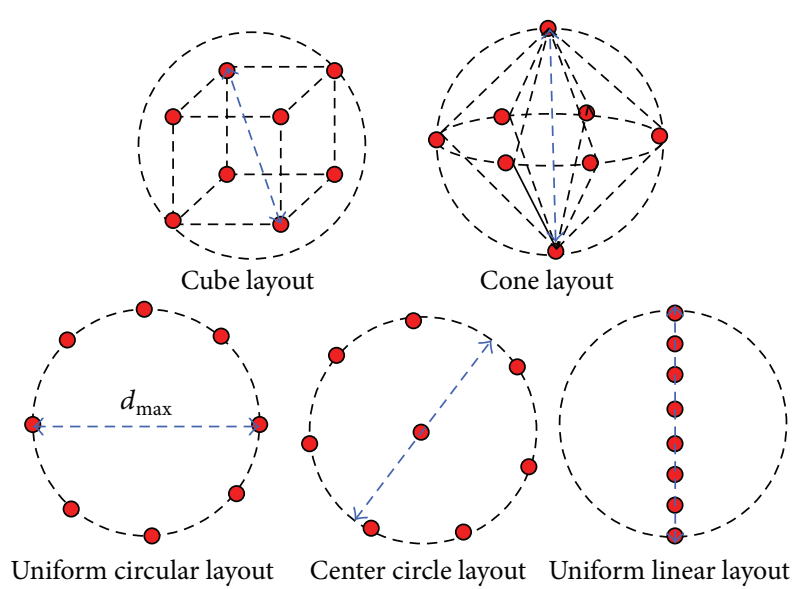

Antenna

FIGURE 2: Reference regular antenna layouts.

Note that (9) is valid only when the eigenvalues $\sigma_{1}, \sigma_{2}, \ldots, \sigma_{L}$ are distinct. However, it can be easily verified that the discontinuous point of $\bar{C}$ at $\sigma_{k} \neq \sigma_{m}, k \neq m$ is the removable discontinuity; that is,

$$
\lim _{\sigma_{k} \rightarrow \sigma_{m}^{+}} \bar{C}=\lim _{\sigma_{k} \rightarrow \sigma_{m}^{-}} \bar{C}
$$

Hence, in case that $\mathbf{R}$ has identical eigenvalues, the right-hand side of (9) can be evaluated with the left or right limits around the discontinuity.

From (9), we know that $\bar{C}$ is a function of $\mathbf{R}$. On the other hand, $\mathbf{R}$ is given by (5). Hence, for fixed PAS and $\mathrm{PES}, \bar{C}$ is completely determined by the antenna layout $\mathbf{P}=$ $\left\{\mathbf{p}_{1}, \mathbf{p}_{2}, \ldots, \mathbf{p}_{L}\right\}$. Our objective is to find the optimal $\mathbf{P}$ which can maximize $\bar{C}$ under the diameter constraint $d_{\max }$. The optimization problem can be formally stated as

$$
\begin{array}{r}
\mathbf{P}^{\text {opt }}=\underset{\mathbf{P} \in \mathbf{R}^{L \times 3}}{\operatorname{argmax}}\{\bar{C}(\mathbf{P})\} \quad \text { s.t. }\left|\mathbf{p}_{l}-\mathbf{p}_{l^{\prime}}\right| \leq d_{\max }, \\
\forall 1 \leq l, l^{\prime} \leq L, l \neq l^{\prime} .
\end{array}
$$

The constraint condition $\left|\mathbf{p}_{l}-\mathbf{p}_{l^{\prime}}\right| \leq d_{\max }$ implies that the antenna elements should be placed within a sphere of diameter $d_{\max }$.

\section{The Optimal Antenna Layout}

The optimization problem shown in (12) is too difficult to be solved directly, so in this section, we use simulated annealing (SA) [24-26] to search for the optimal transmit antenna layout. A brief description on SA and the related SA parameters used in this paper are presented in the Appendix.

We consider the $8 \times 1$ MISO BF system. With $L=8$ antennas, some of the regular antenna placement patterns are illustrated in Figure 2 for reference. 

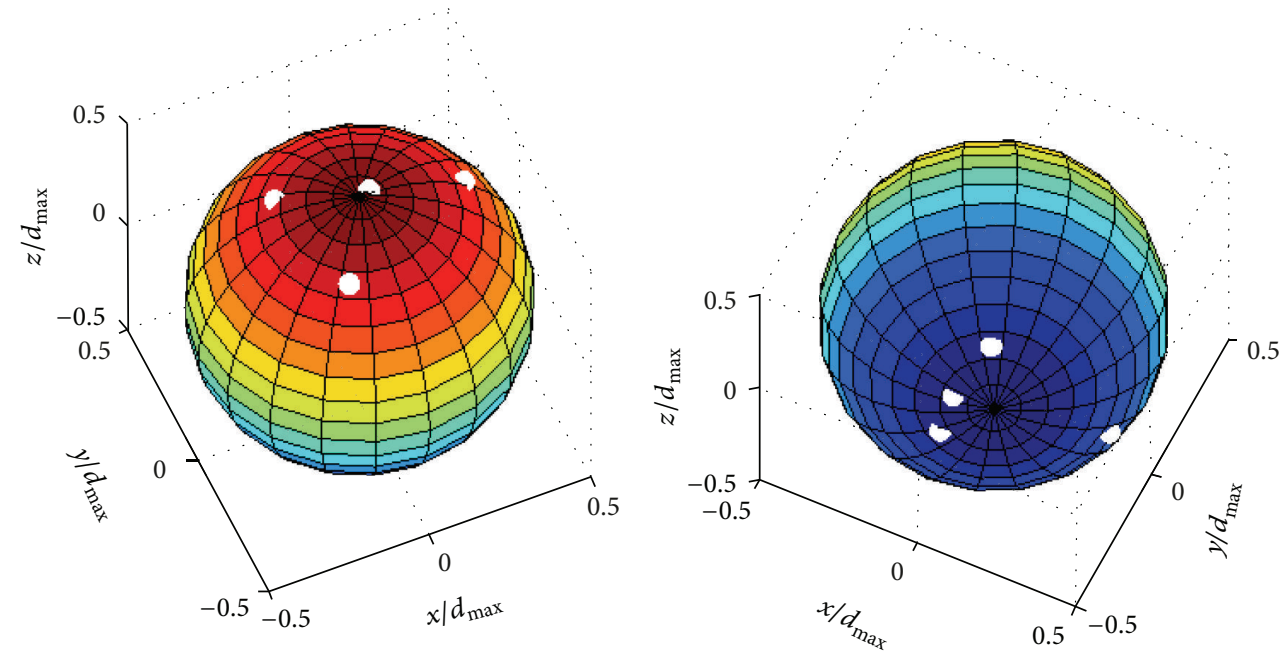

(a) $d_{\max }=0.2 \lambda$
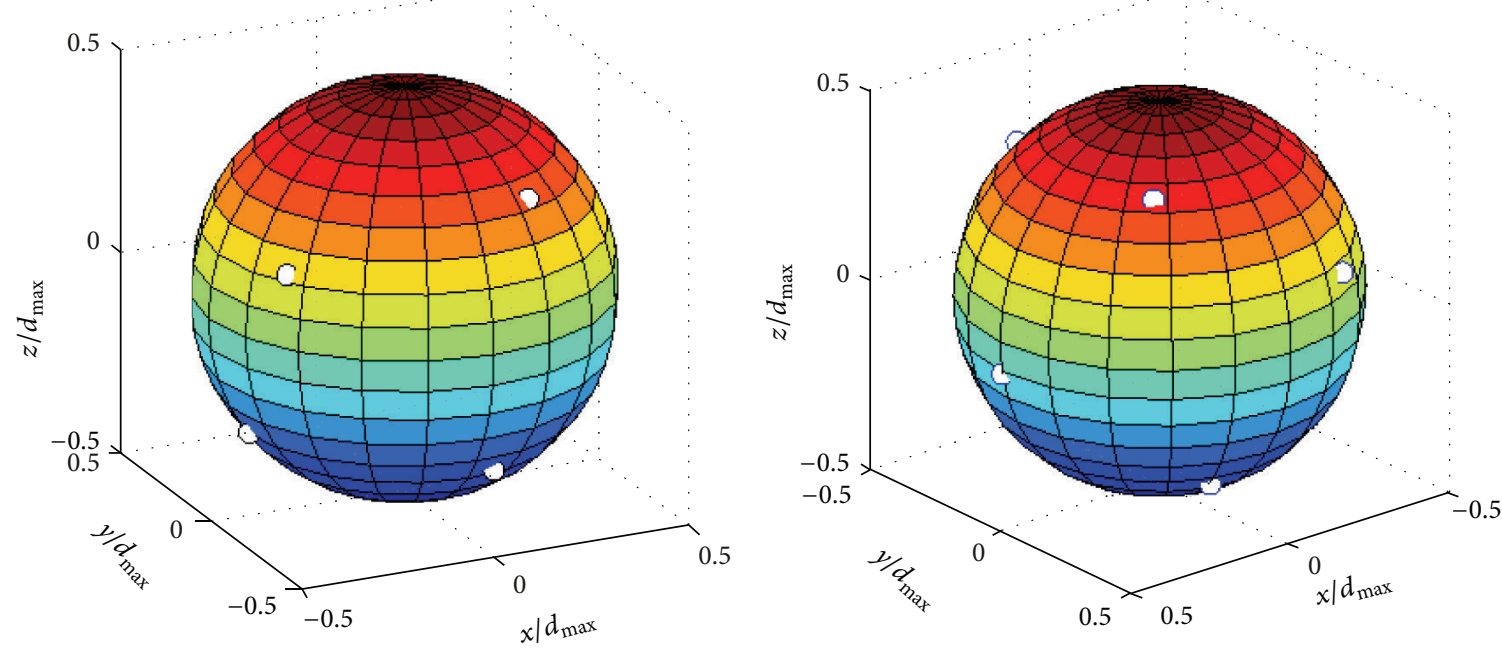

(b) $d_{\max }=0.6 \lambda$
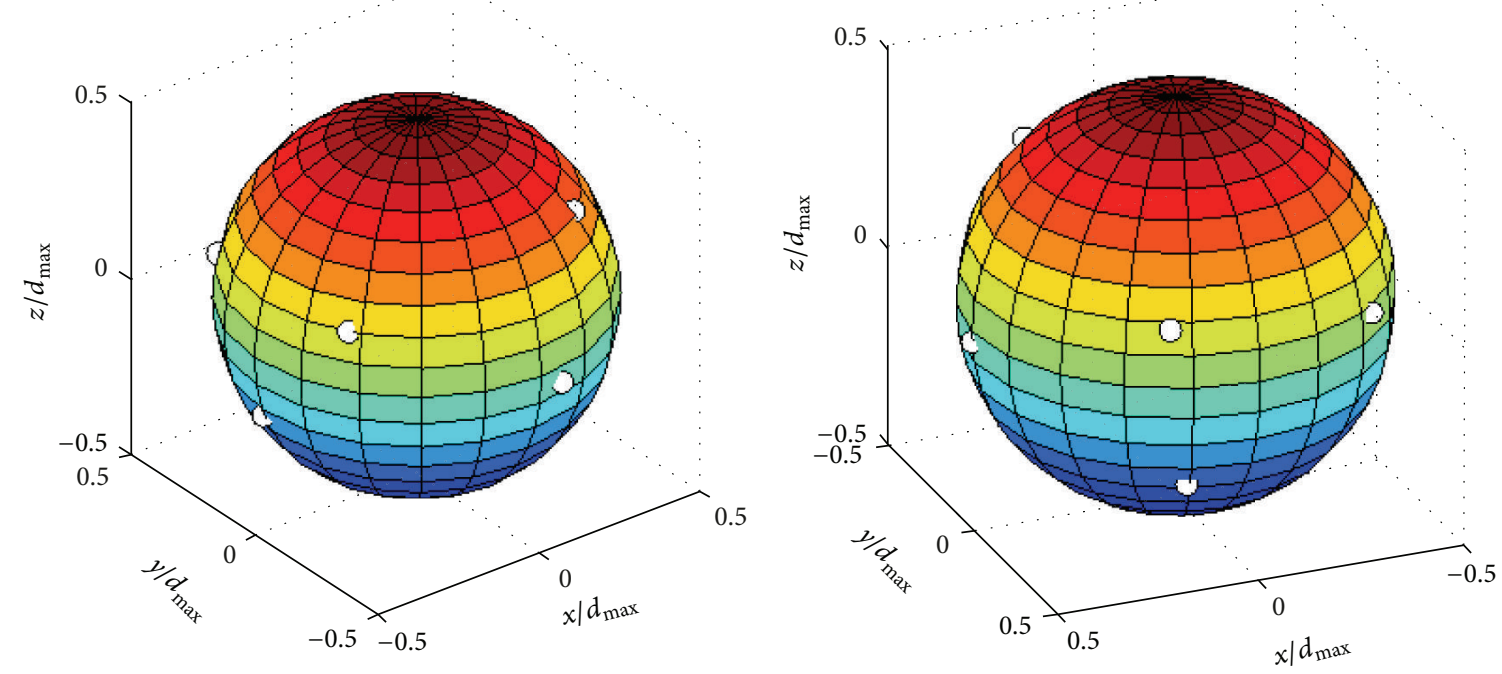

(c) $d_{\max }=\lambda$

FIgURE 3: The optimal layout for Scenario I. 
The optimal antenna layout will certainly depend on the angle spreading of the radio wave. For simplicity, we consider the uniform distribution PAS and PES, which are defined as

$$
\begin{gathered}
P_{e}(\alpha)=\frac{1}{2 \Delta_{\alpha}}\left(\alpha_{0}-\Delta_{\alpha} \leq \alpha \leq \alpha_{0}+\Delta_{\alpha}\right) \\
P_{a}(\beta)=\frac{1}{2 \Delta_{\beta}}\left(\beta_{0}-\Delta_{\beta} \leq \beta \leq \beta_{0}+\Delta_{\beta}\right),
\end{gathered}
$$

where $\Delta_{\alpha}$ and $\Delta_{\beta}$ are the range of $\alpha$ and $\beta \cdot \alpha_{0}$ and $\beta_{0}$ denote the center of angle of departure (AoD). Without loss of generality, we set $\alpha_{0}=\beta_{0}=0$. Substituting (13) into (5), the spatial correlation of transmit antenna is given as

$$
\begin{aligned}
R\left(\mathbf{p}, \mathbf{p}^{\prime}\right)= & \frac{1}{4 \Delta_{\alpha} \Delta_{\beta}} \\
\times \int_{\Delta_{\alpha}}^{\Delta_{\alpha}} \int_{\Delta_{\beta}}^{\Delta_{\beta}} \exp \left\{j \frac{2 \pi}{\lambda}[\right. & \left(x-x^{\prime}\right) \cos \alpha \cos \beta \\
& +\left(y-y^{\prime}\right) \cos \alpha \sin \beta \\
& \left.\left.+\left(z-z^{\prime}\right) \sin \alpha\right]\right\} \mathrm{d} \alpha \mathrm{d} \beta .
\end{aligned}
$$

Five propagation scenarios are considered in this paper, as shown in Table 1. Scenario I is the 3D whole space uniformly scattering environment. In Scenario II, the elevation of the departing rays is compressed to a very narrow domain, the scattering range converges like a $2 \mathrm{D}$ thin plane. Scenario III represents the narrow spreading situation where the angle spreading of departing rays for both azimuth and elevation has been compressed in the $\pm 10^{\circ}$ range. In this case, the departing rays converge like a thin beam. Scenario IV and Scenario V are the degraded cases of Scenario II and Scenario III.

3.1. The Optimal Layouts for Scenario I. Figure 3 shows the optimal antenna layout for Scenario I with diameter constraint as $d_{\max }=0.2 \lambda, d_{\max }=0.6 \lambda$, and $d_{\max }=$ $\lambda$, respectively. For each $d_{\max }$ value, two subfigures are presented which are the two different side views of the same antenna placement layout. An interesting observation is that the optimal antenna layout will always place the antenna elements on the surface of the sphere.

In Figure 4, we compare the ergodic capacity of the optimal layout with that of regular layouts listed in Figure 2. We can see that the cube and the cone layout are near optimal. Specifically, when $d_{\max } \leq 0.4 \lambda$, the ergodic capacity of cube layout is almost the same as that of optimal layout and the cone layout is very close to optimal. When $d_{\max }>0.4 \lambda$, the cone layout is more close to the optimal layout than the cube layout.

3.2. The Optimal Layouts in Scenario II. Figure 5 shows the optimal antenna layout for Scenario II where the angle spread is uniform in the azimuth plane and is narrow in elevation (constrained in $\pm 10^{\circ}$ in elevation plane). Comparing
TABLE 1: PAS and PES for different propagation scenarios.

\begin{tabular}{lcc}
\hline & PES & PAS \\
& $\Delta_{\alpha}$ & $\Delta_{\beta}$ \\
\hline Scenario I & $90^{\circ}$ & $180^{\circ}$ \\
Scenario II & $10^{\circ}$ & $180^{\circ}$ \\
Scenario III & $10^{\circ}$ & $10^{\circ}$ \\
Scenario IV & $0^{\circ}$ & $180^{\circ}$ \\
Scenario V & $0^{\circ}$ & $0^{\circ}$ \\
\hline
\end{tabular}

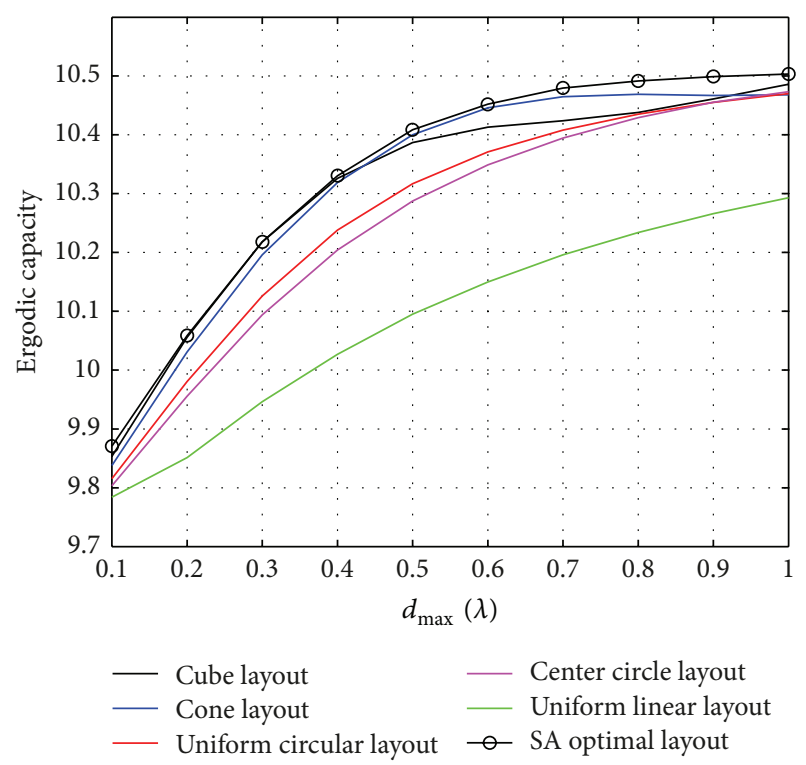

FIGURE 4: Capacity difference between the optimal layout and the reference regular layouts for Scenario I with $\gamma_{0}=20 \mathrm{~dB}$.

Figures 5 and 3, we can see that the angle spread of the departing rays has serious impact on the optimal antenna layout. Since the rays mainly depart from the horizontal plane, the optimal layout is essentially a ring with its normal line pointing in the $z$-direction. Figure 5(b) is an exception where 7 antennas have formed a ring in horizontal plane while the 8th antenna is placed at the norm line of the ring.

If we force the 8th antenna in Figure 5(b) at the norm line of the ring to the center of the horizontal plane ring and keep the positions of the rest 7 antennas, we will arrive at a layout similar to the center-circle layout shown in Figure 2. Figure 6 shows the capacity difference between the layout in Figure 5(b) and the center-circle layout. The results show that the capacity difference is very slight, since the angle spread in elevation is narrow (constrained in $\pm 10^{\circ}$ in elevation plane). In other words, although the optimal layout suggested by SA is Figure 5(b) for $d_{\max }=0.6 \lambda$, which is quite different with Figures 5(a) and 5(c) where the optimal solution is a plane, we know from Figure 6 that forcing the highest point to the $x-y$ plane will lead to almost no loss. What is implicit here is that, with a very narrow angel spreading at elevation, the optimization can focus only on the $x-y$ plane. In fact, we will see later that if the angle spread in elevation is even 


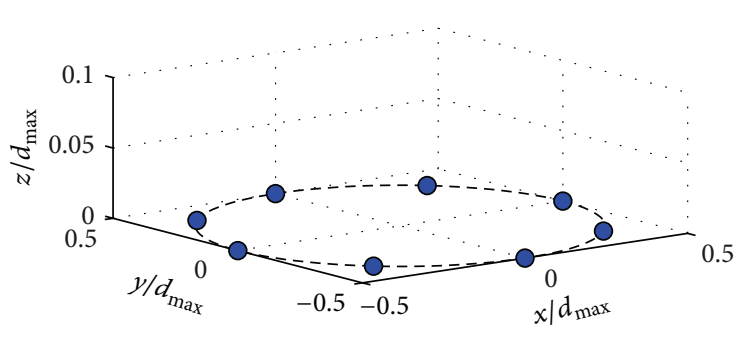

(a) $d_{\max }=0.2 \lambda$

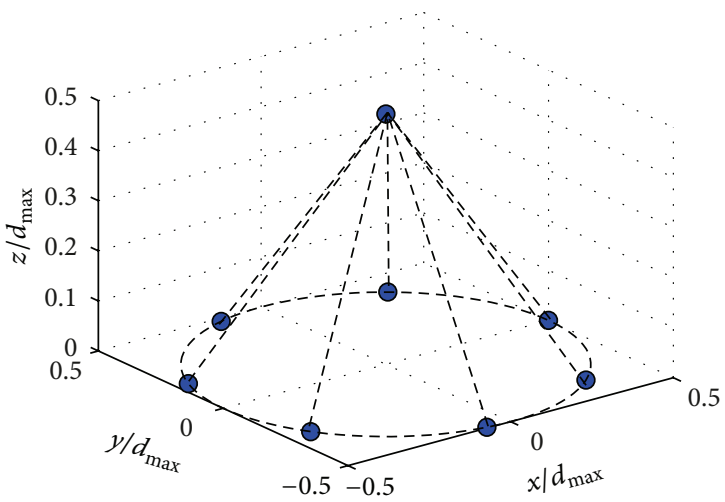

(b) $d_{\max }=0.6 \lambda$

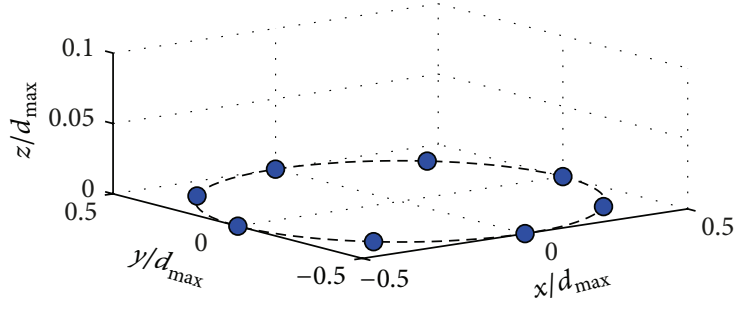

(c) $d_{\max }=\lambda$

FIGURE 5: The optimal layout for Scenario II.

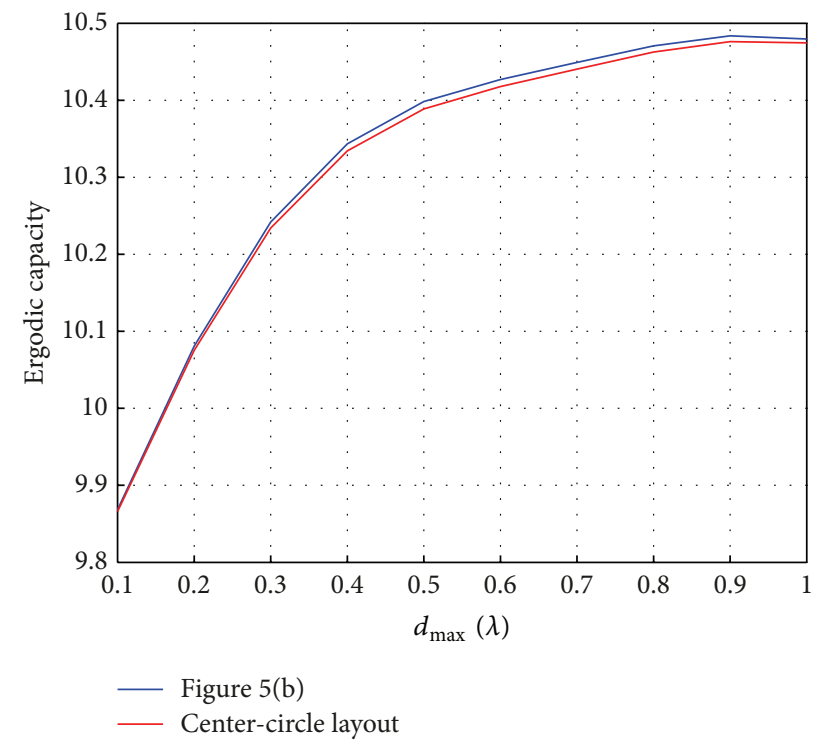

FIGURE 6: Capacity difference between the layout in Figure 5(b) and the corresponding center-circle layout. $\gamma_{0}=20 \mathrm{~dB}$.

smaller as to zero, the $z$-axial coordinate will no more affect the performance.

In Figure 7, we compare the ergodic capacity of the optimal layout under Scenario II with that of regular layouts listed in Figure 2. Unsurprisingly, the uniform circle layout and the center circle layout are near optimal. Specifically, when $d_{\max } \leq 0.5 \lambda$, the ergodic capacity of the uniform circle layout is almost the same as that of optimal layout. When

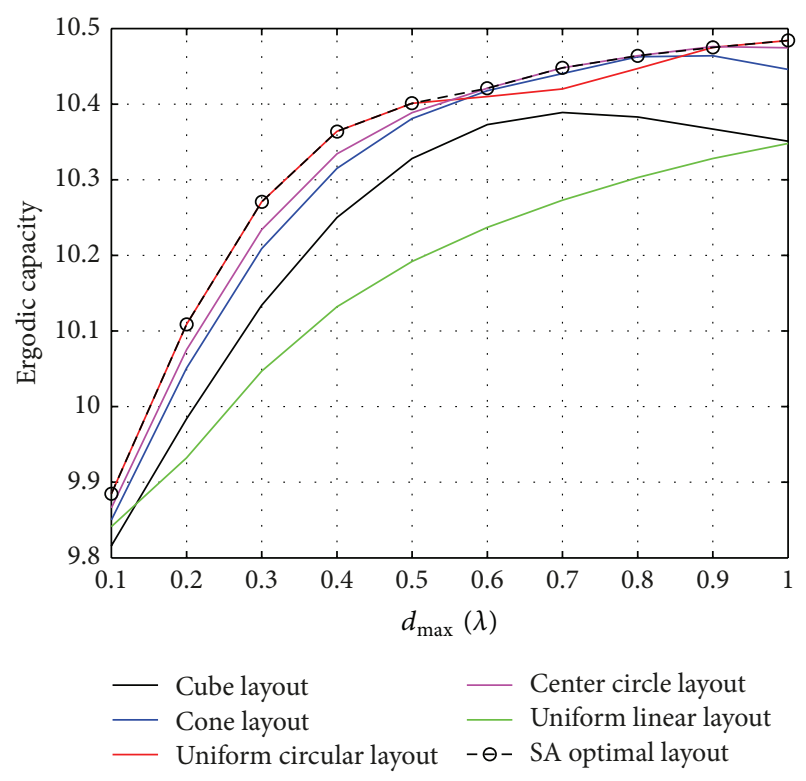

FIGURE 7: Capacity difference between the optimal layout and the reference regular layouts for Scenario II. $\gamma_{0}=20 \mathrm{~dB}$.

$d_{\max }>0.5 \lambda$, the center circle layout is more close to the optimal layout than the uniform circle layout.

3.3. The Optimal Layouts in Scenario III. Figure 8 shows the optimal antenna layout for Scenario III where the angle spread is narrow in both azimuth and elevation (constrained in $\pm 10^{\circ}$ in azimuth and elevation plane). Comparing 


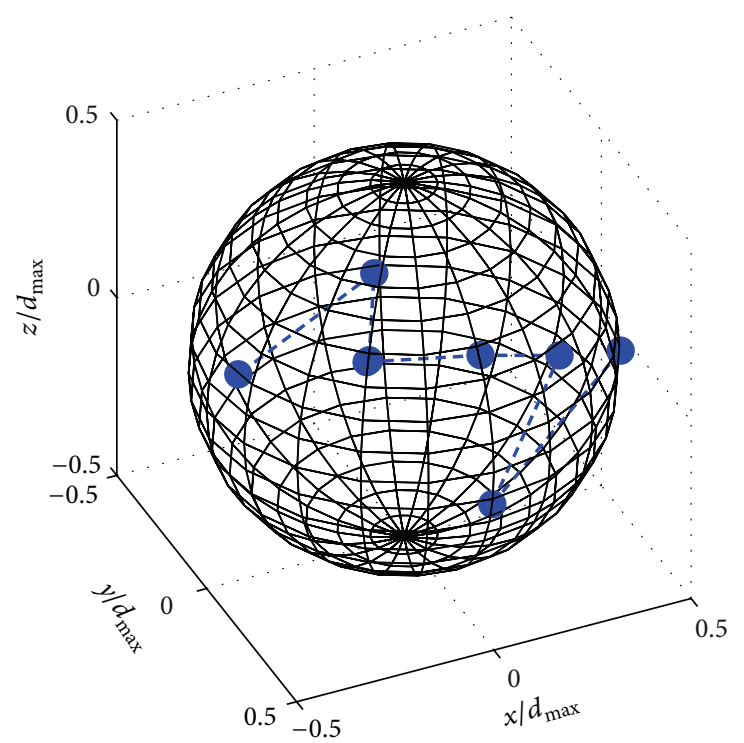

(a) $d_{\max }=0.2 \lambda$

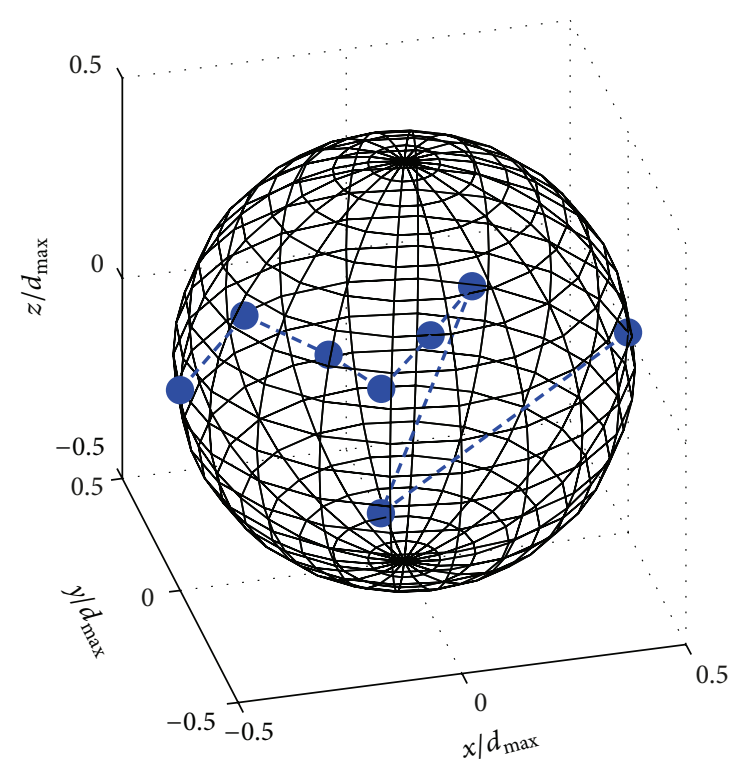

(b) $d_{\max }=0.6 \lambda$

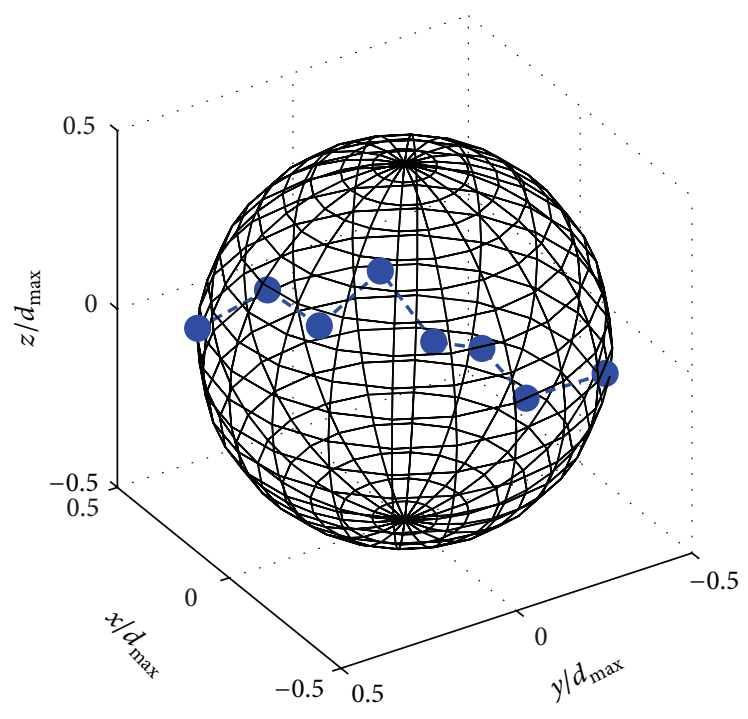

(c) $d_{\max }=\lambda$

FIGURE 8: The optimal layout for Scenario III.

Figures 8,5, and 3, we can see that the optimal antenna layout dimension is highly dependent on the power azimuth spectrum (PAS) distribution and power elevation spectrum (PES) distribution. In this case, the departing rays converge to a thin beam which is approximately a single plane wave. The optimal layout in this case is different with all the results in the two scenarios mentioned previously, the antennas no longer distribute on the sphere surface. The eight antennas are some like eight nodes on a knotted rope, but each of them has its own vibration amplitude and these amplitudes are irregular.

In Figure 9, we compare the ergodic capacity of the optimal layout under Scenario III with that of regular layouts listed in Figure 2. We can see that when the angle spreading is very narrow both in elevation and azimuth, the uniform linear array is the one closet to the optimal, among the layouts listed in Figure 2.

3.4. The Optimal Layouts in Scenario IV. Scenario IV is the degraded case of Scenario II. The range of PES has been further compressed by setting $\Delta_{\alpha}$ in (13) to zero, resulting in that the $P_{e}(\alpha)$ becomes the Dirac impulse function and the double integral in (14) simplifies to a single integral as shown in (15). The $z$-axial coordinate disappears from (15) implying that the $z$-axial coordinate will no more affect the antenna spatial correlation. The $3 \mathrm{D}$ antenna layout optimization problem is then reduced to a $2 \mathrm{D}$ optimization problem. With 


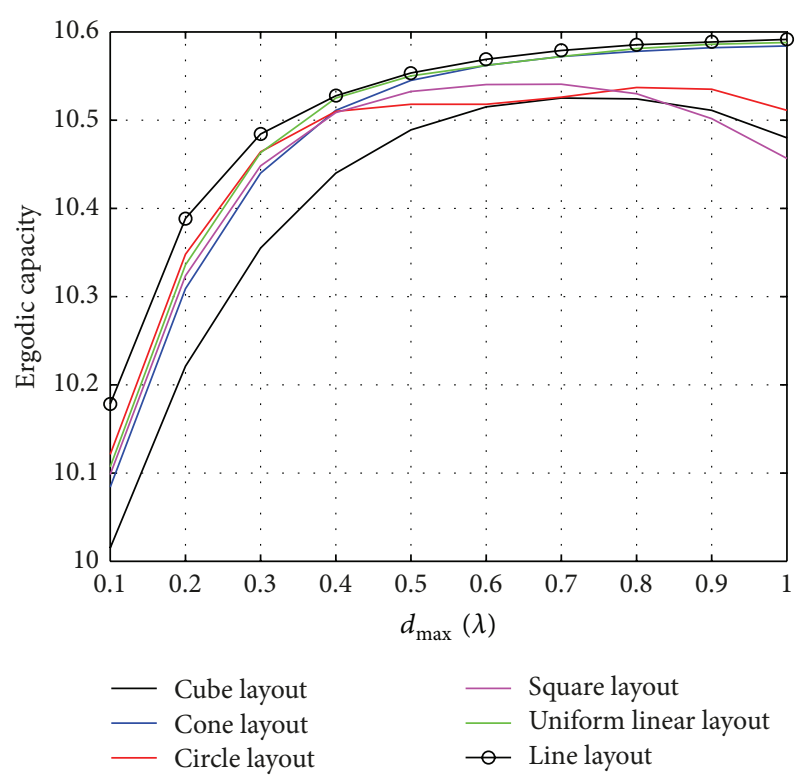

FIGURE 9: Capacity difference between the optimal layout and the reference regular layouts for Scenario III. $\gamma_{0}=20 \mathrm{~dB}$.

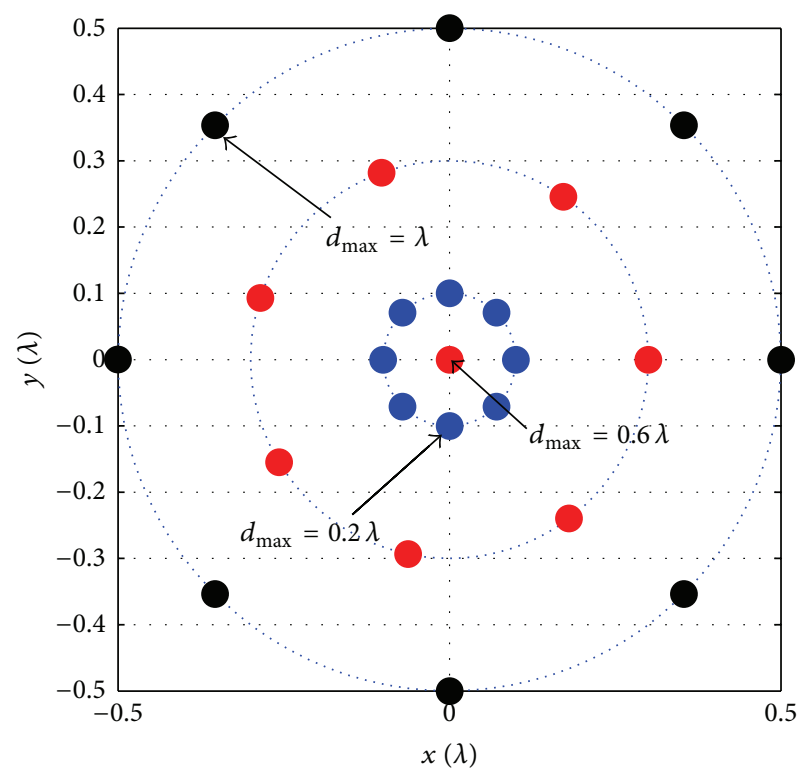

FIGURE 10: The optimal layout for Scenario IV with various $d_{\max }$.

a constraint that the antenna elements must be placed within a circle of diameter $d_{\max }$ :

$$
\begin{aligned}
R\left(\mathbf{p}, \mathbf{p}^{\prime}\right) & \\
=\frac{1}{2 \Delta_{\beta}} \int_{\Delta_{\beta}}^{\Delta_{\beta}} \exp \left\{j \frac{2 \pi}{\lambda}\right. & {\left[\left(x-x^{\prime}\right) \cos \beta\right.} \\
& \left.\left.+\left(y-y^{\prime}\right) \sin \beta\right]\right\} \mathrm{d} \beta .
\end{aligned}
$$

Figure 10 shows the optimal antenna coordinates for Scenario IV which is the output of SA. The points in red, blue

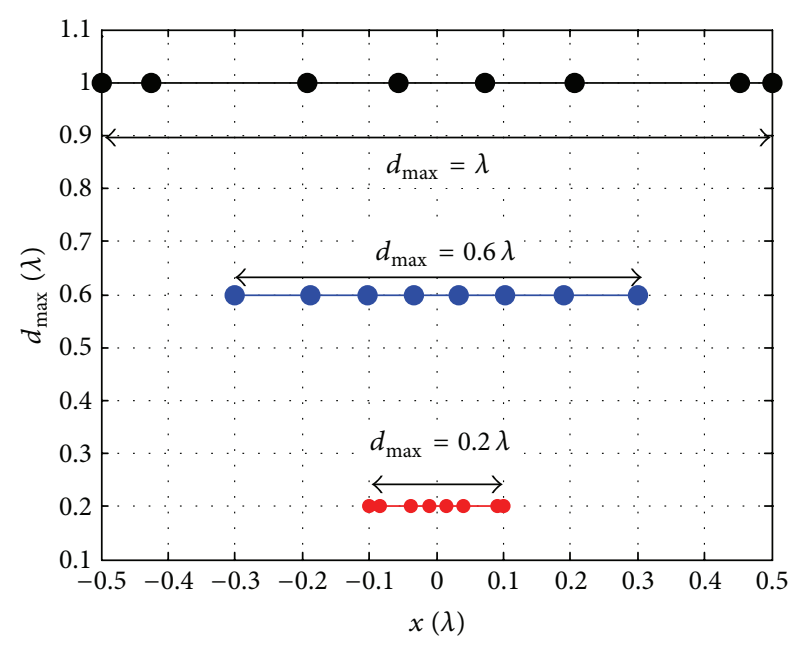

FIgURE 11: Optimal layout for Scenario $\mathrm{V}$ with various $d_{\max }$.

or black denote, respectively, the optimal antenna locations for different $d_{\max }$ constraints. In case that $d_{\max }=0.2 \lambda$ and $d_{\max }=\lambda$, the antennas are almost uniformly distributed on a circle while in the case of $d_{\max }=0.6 \lambda$, one antenna is placed at the center of the circle and the rests are nonuniformly distributed on the circle.

3.5. The Optimal Layouts in Scenario V. Scenario V is the degraded case with both $\Delta_{\alpha}$ and $\Delta_{\beta}$ set to zero. Now the double integral in (14) disappears and it reduces to (16). This indicates that the optimal layout does not depend on $z$-axial and $y$-axial coordinates and the original 3D antenna layout optimization problem is reduced to a $1 \mathrm{D}$ optimization problem with a constraint that all antennas should be placed in a interval of length $d_{\max }$ :

$$
R\left(\mathbf{p}, \mathbf{p}^{\prime}\right)=\exp \left[j \frac{2 \pi}{\lambda}\left(x-x^{\prime}\right)\right]
$$

Figure 11 is the optimal layout obtained through SA. The points in red, blue, or black correspond to three difference $d_{\max }$ constraints. We can see that the optimal layout in this case is the nonuniform linear array.

In summary, in the three-dimensional (3D) space uniformly scattering environment, the optimal antenna layout will locate the antenna elements on the surface of a sphere. For practical consideration, the cube layout is a good choice for $d_{\max } \leq 0.4 \lambda$ and the cone layout is better for $d_{\max }>0.4 \lambda$. When the scattering is concentrated in a two-dimensional (2D) plane, the circular layout is the optimal. For practical consideration, we may consider the uniform circular layout for $d_{\max } \leq 0.5 \lambda$ and the center circle layout for $d_{\max }>0.5 \lambda$. While the scattering is very narrow in both elevation and azimuth, the optimal layout is nearly a nonuniform linear array. The uniform linear layout is near optimal particularly when $d_{\max }>0.4 \lambda$. 
(1) Generate a random initial configuration of antennas $\mathbf{P}_{\mathbf{0}}$, and $\mathbf{P}^{\text {opt }}=\mathbf{P}_{\mathbf{0}}$, compute the cost function value $f\left(\mathbf{P}_{0}\right)$;

(2) Set the initial temperature $T(0)=T_{0}$ and the iterations $i=1$;

(3) Do while $T(i)>T_{\text {end }}$

(1) for inner loop $m=1 \sim K$

(2) Do some small random perturbation to the $\mathbf{P}^{\text {opt }}$ that yields a nearby solution $\mathbf{P}_{\text {new }}$. Compute $f\left(\mathbf{P}_{\text {new }}\right)$ and $\Delta f=f\left(\mathbf{P}_{\text {new }}\right)-f\left(\mathbf{P}^{\text {opt }}\right)$

(3) if $\Delta f \leq 0, \mathbf{P}^{\text {opt }}=\mathbf{P}_{\text {new }}$;

(4) if $\Delta f>0, u=\exp (-\Delta f / T(i))$; generate a random variable $c=\operatorname{random}[0,1]$, if $c<u, \mathbf{P}^{\text {opt }}=\mathbf{P}_{\text {new }}$.

(5) end for

(4) $i=i+1$;

(5) $T(i+1)=T(i)$;

(6) End Do while

(7) Output $\mathbf{P}^{\text {opt }}$

Algorithm 1: Process of SA algorithms.

\section{Conclusions}

This paper investigates the problem of arranging the transmit antennas to attain the maximum ergodic capacity in an MISO beamforming system. Since the optimization problem cannot be solved analytically, we use simulated annealing (SA) algorithm to search for the optimal antenna layout. The results show that the optimal antenna layout dimension is highly dependent on the power azimuth spectrum (PAS) distribution and power elevation spectrum (PES) distribution.

\section{Appendix}

Simulated annealing [24-26] is a technique for combinatorial optimization problems, such as minimizing functions of many variables. For our purposes, a combinatorial optimization problem is one in which we seek to find some configurations of antenna layout $\mathbf{P}=\left\{\mathbf{p}_{1}, \mathbf{p}_{2}, \ldots, \mathbf{p}_{L}\right\}$ in (12) that minimizes the $f(\mathbf{P})=-\bar{C}$ in (9), which is defined as the cost or objective function of SA in this paper. SA starts with a random or feasible initial solution and moves step by step towards a solution $\mathbf{S}^{\text {opt }}$ giving hopefully the minimum (or close to the minimum) of the cost function.

When the configuration of antenna layout $\mathbf{P}=\left\{\mathbf{p}_{1}, \mathbf{p}_{2}\right.$, $\left.\ldots, \mathbf{p}_{L}\right\}$ is at a feasible solution, we attempt some small random perturbation to the configuration that yields a nearby solution. This process can continue starting from the new configuration until no further improvements are obtained, at which point the process terminates. This strategy seems reasonable, but it has a serious problem: it is easily trapped in local minimum, solutions that look good in some small neighborhood of the cost surface but are not necessarily the global optimum. To avoid this problem, the acceptance function is used to decide the acceptance probability.

The probability of accepting a move that causes an increase $\Delta f$ in $f(\mathbf{P})=-\bar{C}$ is determined by the acceptance function. Like most researchers, we use the Metropolis acceptance function [24] $\exp (-\Delta f / T)$, where $T$ is a control parameter corresponding to the temperature in analogy with physical annealing. Because $\Delta f>0$ and $T>0, \exp (-\Delta f / T)$ is always smaller than 1 . We generate a random number
TABLE 2: Parameters for SA algorithms.

\begin{tabular}{lc}
\hline Cost function & $-\bar{C}$ in $(9)$ \\
Initial temperature & $T_{0}=100 ;$ \\
Cooling schedule & $T(t)=0.9 T(t-1)$ \\
Ending temperature & $T_{\text {end }}=0.0001$ \\
Inner loop & $K=100$ \\
\hline
\end{tabular}

$c$ uniformly distributed on the interval $[0,1]$ and compare $\exp (-\Delta f / T)$ with $c$. If $\exp (-\Delta f / T)>a$, then the move is accepted; otherwise, the move is rejected.

The initial temperature $T_{0}$ is set to be 100 , and the ending temperature $T_{\text {end }}$ equals 0.0001 . The cooling schedule will be the simplest possible: $T_{\text {new }}=b T_{\text {old }}, 0<b<1$, where the initial temperature and cooling rate $b$ are determined empirically to give good results and we let $b$ be 0.9 in this paper. At each temperature, we perform 100 moves. The stopping criterion is to terminate annealing when the cost improvement seen across five successive temperatures or the temperature is cooler than the ending temperature. The key SA parameters in this paper are listed in Table 2 and the detailed process of SA is shown in Algorithm 1.

\section{Acknowledgment}

This paper was supported by National Natural Science Foundation of China (no. 61072059).

\section{References}

[1] G. J. Foschini and M. J. Gans, "On limits of wireless communications in a fading environment when using multiple antennas," Wireless Personal Communications, vol. 6, no. 3, pp. 311-335, 1998.

[2] J. H. Winters, "On the capacity of radio communication systems with diversity in Rayleigh fading environments," IEEE Journal on Selected Areas in Communications, vol. 5, no. 5, pp. 871-878, 1987.

[3] T. K. Y. Lo, "Maximum ratio transmission," IEEE Transactions on Communications, vol. 47, no. 10, pp. 1458-1461, 1999. 
[4] W. Li, X. Huang, and H. Leung, "Performance evaluation of digital beamforming strategies for satellite communications," IEEE Transactions on Aerospace and Electronic Systems, vol. 40, no. 1, pp. 12-26, 2004.

[5] Y. Yang, B. Bai, W. Chen et al., "A low-complexity crosslayer algorithm for coordinated downlink scheduling and Robust beamforming under a limited feedback constraint," IEEE Transactions on Vehicular Technology, no. 99, pp. 1-13, 2013.

[6] P. A. Dighe, R. K. Mallik, and S. S. Jamuar, "Analysis of transmitreceive diversity in Rayleigh fading," IEEE Transactions on Communications, vol. 51, no. 4, pp. 694-703, 2003.

[7] J. Choi, "Opportunistic beamforming with single beamforming matrix for virtual antenna arrays," IEEE Transactions on Vehicular Technology, vol. 60, no. 3, pp. 872-881, 2011.

[8] S. Lee, S. Moon, H. Kong, and I. Lee, "Optimal beamforming schemes and its capacity behavior for downlink distributed antenna systems," IEEE Transactions on Communications, vol. 99, pp. 1-10, 2013.

[9] A. Maaref and S. Aissa, "Closed-form expressions for the outage and ergodic shannon capacity of MIMO MRC systems," IEEE Transactions on Communications, vol. 53, no. 7, pp. 1092-1095, 2005.

[10] A. Zanella, M. Chiani, and M. Z. Win, "Performance of MIMO MRC in correlated Rayleigh fading environments," in Proceedings of the IEEE Vehicular Technology Conference (VTC '05), vol. 3, pp. 1633-1637, Stockholm, Sweden, June 2005.

[11] M. R. McKay, I. B. Collings, and P. J. Smith, "Capacity and ser analysis of MIMO beamforming with MRC," in Proceedings of the IEEE International Conference on Communications, vol. 3, pp. 1326-1330, June 2006.

[12] M. T. Ivrlac, W. Utschick, and J. A. Nossek, "Fading correlations in wireless MIMO communication systems," IEEE Journal on Selected Areas in Communications, vol. 21, no. 5, pp. 819-828, 2003.

[13] M. di Renzo and H. Haas, "Bit error probability of SMMIMO over generalized fading channels," IEEE Transactions on Vehicular Technology, vol. 61, no. 3, pp. 1124-1144, 2012.

[14] R. Nordin and M. Ismail, "Impact of spatial correlation towards the performance of MIMO downlink transmissions," in Proceedings of the 18th Asia Pacific Conference on Communications (APCC '12), pp. 390-395, October 2012.

[15] G. Singh, P. Mishra, and R. Vij, "Performance evaluation of MLVBLAST MIMO decoder using different antenna configuration using rican and Rayleigh channel," in Proceedings of the IEEE Conference on Communication Systems and Network Technologies (CSNT '13), pp. 174-179, April 2013.

[16] J.-A. Tsai, R. M. Buehrer, and B. D. Woerner, "BER performance of a uniform circular array versus a uniform linear array in a mobile radio environment," IEEE Transactions on Wireless Communications, vol. 3, no. 3, pp. 695-700, 2004.

[17] Y. Masoudi, S. Lotfi, E. Laleh, and F. Fathy, "Mobile antenna placement using combination of genetic algorithm and Learning automata," in Proceedings of the IEEE Conference on Communication Systems and Network Technologies (CSNT '13), pp. 22-28, April 2013.

[18] P. Uthansakul, D. Assanuk, and M. Uthansakul, "The use of genetic algorithm for designing MIMO antenna placement," in Proceedings of the International Conference on Computer and Information Application (ICCIA '10), pp. 414-417, December 2010.

[19] Q. Wang, H. Yang, and L.-Y. Dai, "MIMO capacity with different antenna layout," in Proceedings of the 2nd International
Conference on Networks Security, Wireless Communications and Trusted Computing (NSWCTC'10), vol. 1, pp. 45-48, April 2010.

[20] Q. Wang and H. Yang, "Optimal antenna layout under uniformly scattering environments," in Proceedings of the International Conference on Wireless Communications and Signal Processing (WCSP '10), pp. 1-5, October 2010.

[21] X. Pu, S. Shao, and Y. Tang, "Optimal $2 \times 2$ antenna placement for short-range communications," IEEE Communications Letters, vol. 17, no. 8, pp. 1560-1563, 2013.

[22] S. Kaul, K. Ramachandran, P. Shankar et al., "Effect of antenna placement and diversity on vehicular network communications," in Proceedings of the 4th Annual IEEE Communications Society Conference on Sensor, Mesh and Ad Hoc Communications and Networks (SECON '07), pp. 112-121, June 2007.

[23] W. Fan, F. Sun, and P. Kyösti, "3D channel emulation in multiprobe setup," Electronics Letters, vol. 49, pp. 623-625, 2013.

[24] R. A. Rntenbar, "Simulated annealing algorithms: an overview," IEEE Circuits and Devices Magazine, vol. 5, no. 1, pp. 19-26, 1989.

[25] Q. Ji-Yang, "Application of improved simulated annealing algorithm in facility layout design," in Proceedings of the 29th Chinese Control Conference (CCC '10), pp. 5224-5227, July 2010.

[26] J. Tian and M. Gao, "Soft measurement modeling based on improved simulated annealing neural network for sewage treatment," in Proceedings of the WRI World Congress on Software Engineering (WCSE '09), vol. 4, pp. 486-489, May 2009.

[27] W. Yue-yu and G. Li-li, "Spatial correlation in threedimensional receiver antenna array channel model," Computer Engineering, vol. 34, no. 12, pp. 9-12, 2008 (Chinese).

[28] R. H. Clarke, "A statistical theory of mobile-radio reception," The Bell System Technical Journal, vol. 47, pp. 957-1000, 1968.

[29] W. C. Jakes, Microwave Mobile Communication, John Wiley \& Sons, New York, NY, USA, 1974.

[30] T. L. Fulghum, K. J. Molnar, and A. Duel-Hallen, “The Jakes fading model for antenna arrays incorporating azimuth spread," IEEE Transactions on Vehicular Technology, vol. 51, no. 5, pp. 968-977, 2002.

[31] A. Goldsmith, Wireless Communications, Cambridge University Press, New York, NY, USA, 2004.

[32] R. K. Mallik and M. Z. Win, "Channel capacity of adaptive transmission with maximal ratio combining in correlated Rayleigh fading," IEEE Transactions on Wireless Communications, vol. 3, no. 4, pp. 1124-1133, 2004.

[33] M. Kang and M.-S. Alouini, "Impact of correlation on the capacity of MIMO channels," in Proceedings of the International Conference on Communications (ICC '03), vol. 4, pp. 2623-2627, May 2003. 

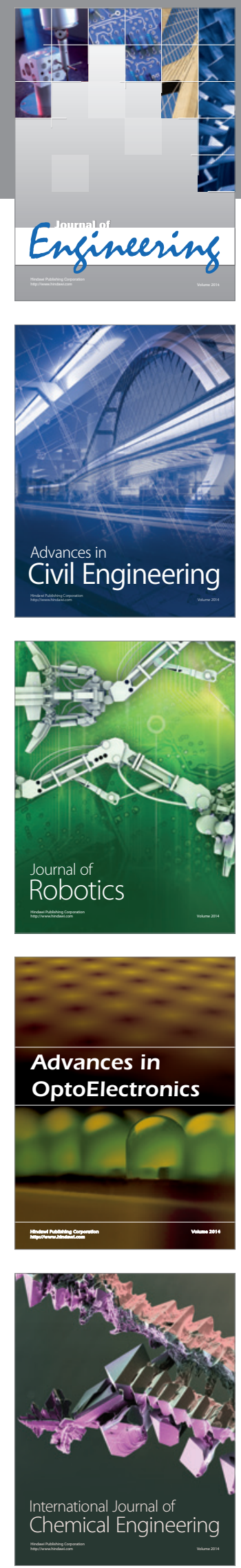

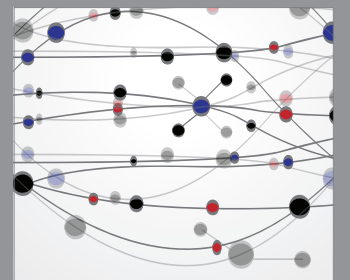

The Scientific World Journal
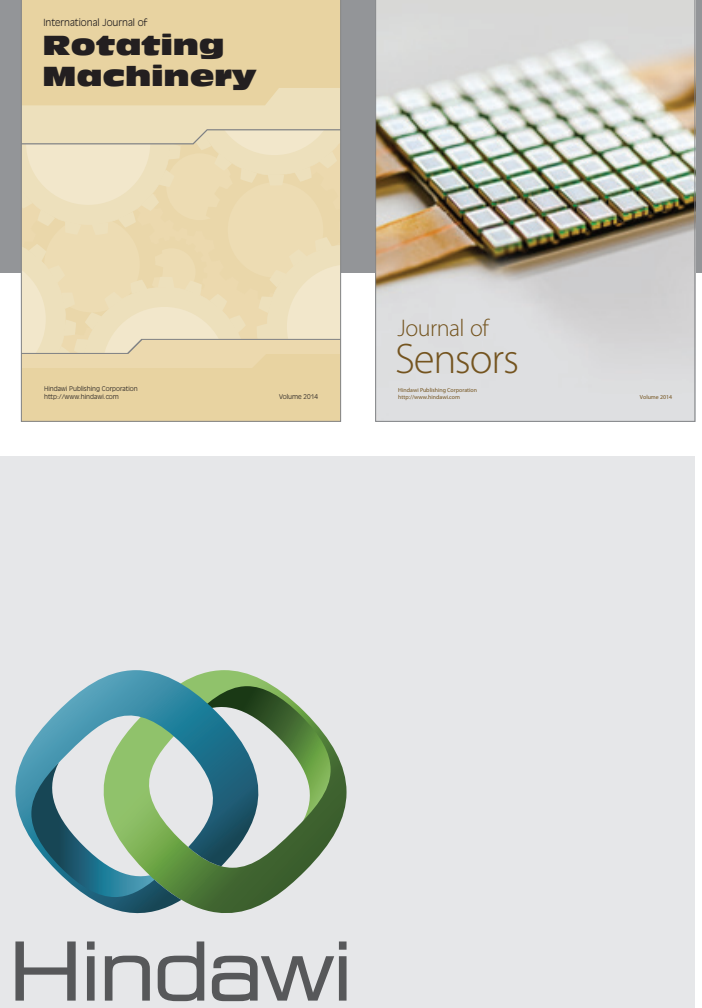

Submit your manuscripts at http://www.hindawi.com
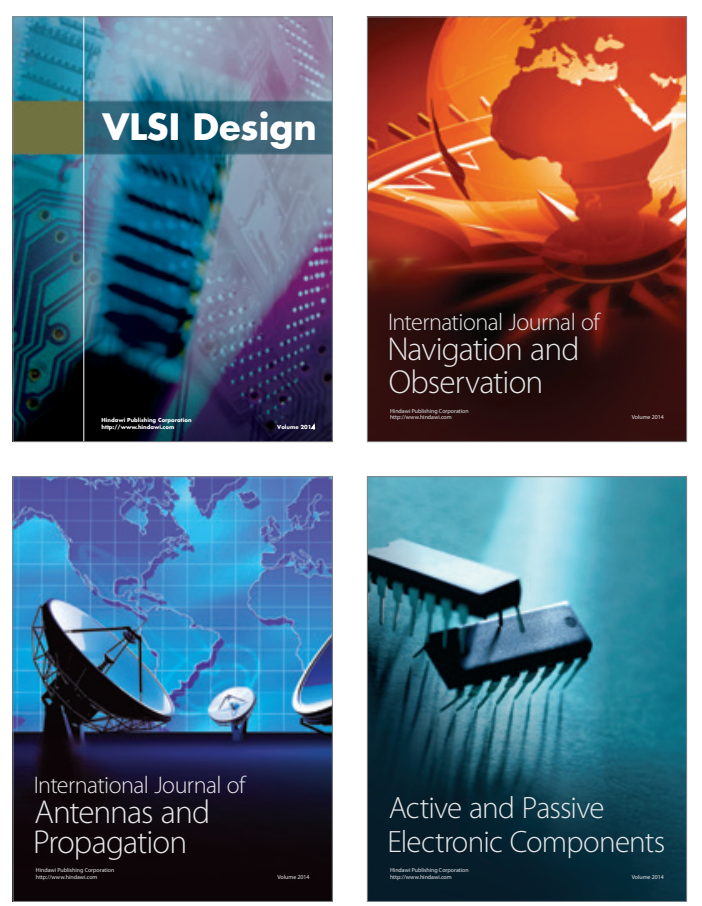
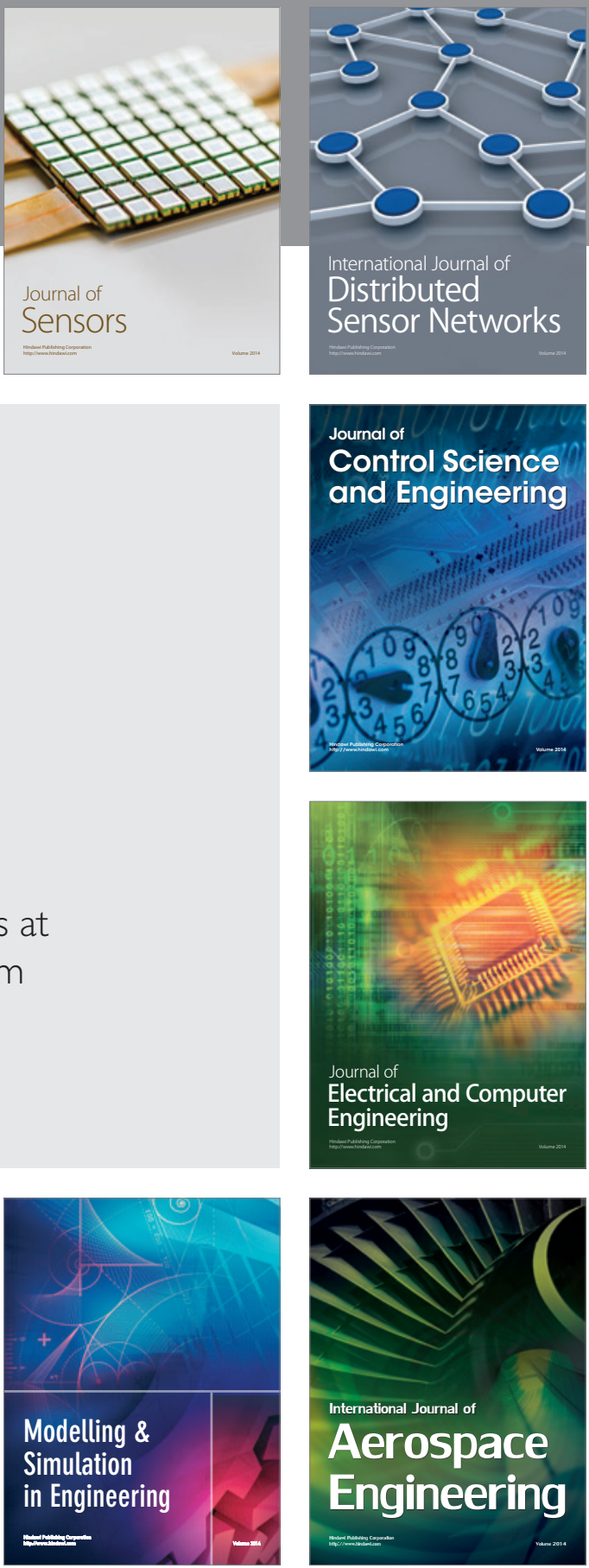

Journal of

Control Science

and Engineering
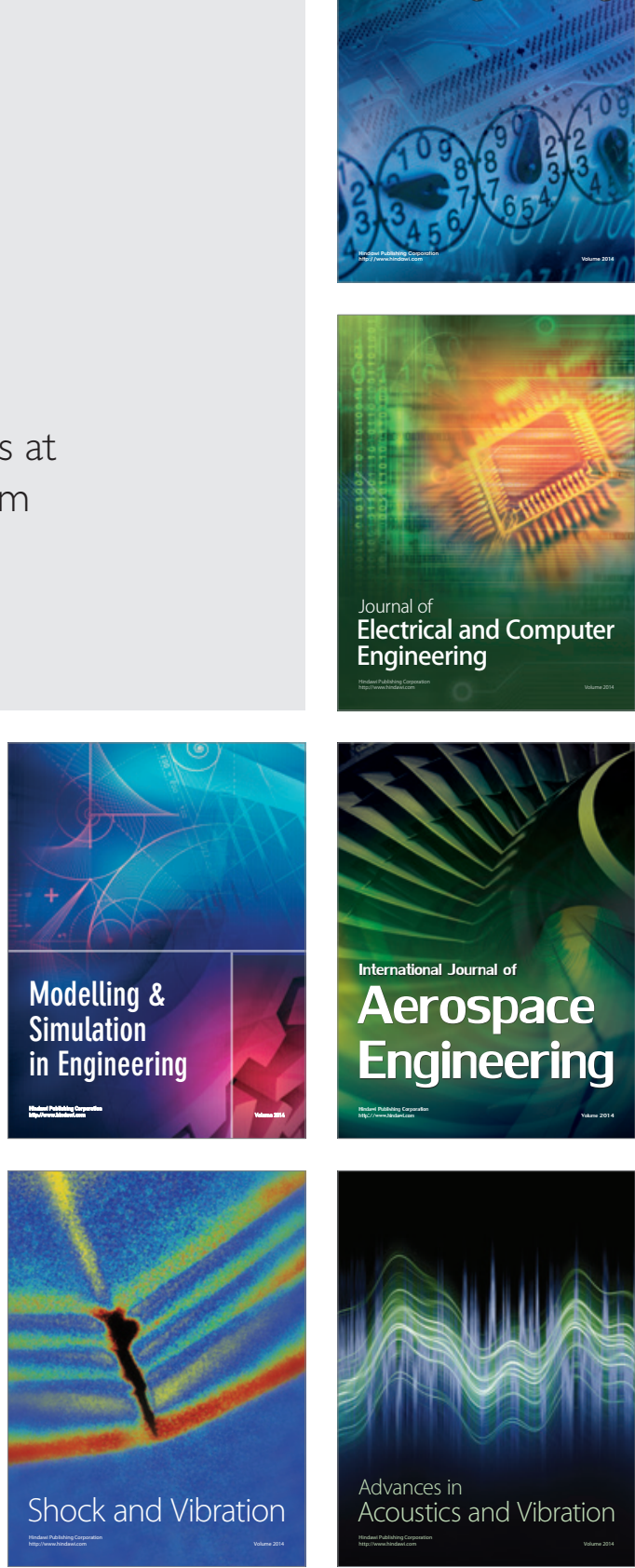